\title{
Stick-slip substructure in rapid tape peeling
}

\author{
S. T. Thoroddsen, ${ }^{1}$ H. D. Nguyen, ${ }^{2}$ K. Takehara, ${ }^{2}$ and T. G. Etoh ${ }^{2}$ \\ ${ }^{1}$ Division of Physical Sciences and Engineering, King Abdullah University of Science and Technology, Thuwal 23955-6900, Saudi Arabia \\ ${ }^{2}$ Civil and Environmental Engineering, Kinki University, Higashi-Osaka 577-8502, Japan \\ (Received 9 April 2010; revised manuscript received 16 July 2010; published 15 October 2010)
}

\begin{abstract}
The peeling of adhesive tape is known to proceed with a stick-slip mechanism and produces a characteristic ripping sound. The peeling also produces light and when peeled in a vacuum, even X-rays have been observed, whose emissions are correlated with the slip events. Here we present direct imaging of the detachment zone when Scotch tape is peeled off at high speed from a solid surface, revealing a highly regular substructure, during the slip phase. The typical 4-mm-long slip region has a regular substructure of transverse $220 \mu \mathrm{m}$ wide slip bands, which fracture sideways at speeds over $300 \mathrm{~m} / \mathrm{s}$. The fracture tip emits waves into the detached section of the tape at $\sim 100 \mathrm{~m} / \mathrm{s}$, which promotes the sound, so characteristic of this phenomenon.
\end{abstract}

PACS number(s): 62.20.M-, 83.60.Uv, 68.35.Np, 82.35.Gh

The peeling of adhesive polymer, or other soft materials, from a solid surface, is of fundamental interest in fracture mechanics [1-6], but also arises in many methods used for pattern fabrication. Manufacturing of surface patterns, even on the micro- or nanoscales, often involves a fabrication step, where a soft material is peeled away from a solid template. This is becoming more prevalent with the fabrication of nanostuctures and flexible electronics. Tape peeling has received considerable theoretical treatment and represents dynamics at the boundary between solid and liquid fracture $[7,8]$. The related phenomenon of triboluminescence is of great interest, as it can generate X-rays, in ways not fully understood [9].

Direct observations of high-speed peeling are challenging as they involve a combination of small length scales and very fast fracture dynamics. Recent observations of Cortet et al. [10] have allowed the construction of time-space diagrams to reveal new aspects of the front motion and identify characteristic frequencies of the stick-slip regime, using video frame rates up to $16 \mathrm{kfps}$. Herein we exploit an ultrahigh-speed video camera [11] at $1000000 \mathrm{fps}$ to directly observe the peeling front.

The tape was slowly pulled from a fresh roll and spread evenly onto either a glass microscope slide or a thick acrylic plate and subsequently pulled manually from the surface at rapid speed. Figure 1(a) shows the experimental setup used. The pulling angle $\theta$, measured between substrate and the detached tape, reduces slightly $\left(<2^{\circ}\right)$ as the tape is pulled off, but was in most cases around $45^{\circ}$ in the region of observation. The tape slides on a horizontal rod to keep $\theta$ constant between experiments. Most experiments used a roll of 18mm-wide 3M Scotch Tape, but results were identical for a 12 $\mathrm{mm}$ roll. The tape material consists of sticky acrylic adhesive on an acetate film, with a total thickness of $58 \mu \mathrm{m}$.

To capture the rapid peeling motions, we use an ultrahigh-speed CCD video camera (Shimadzu Hypervision), see Etoh et al. [11]. Each video sequence consists of 102 frames, containing $260 \times 312$ pixels, irrespective of the frame rate used, thus allowing for good spatial resolution, even at the highest frame rates. The tape itself acts as a diffuser for direct lighting from a metal halide lamp (Sumita LS-M350), which is imaged with a long-distance microscope, with magnification up to 4.6. Triggering was accomplished by the detached part of the tape blocking a laser beam.
Figure 1(b) shows the overall stick-slip structure including the thin fracture bands, which occur during the slip phase. In our experiments the detachment front is pulled at a high average velocity of $4-14 \mathrm{~m} / \mathrm{s}$, with the forward movement in the slip phase proceeding at about twice this speed, i.e., in the range $9-27 \mathrm{~m} / \mathrm{s}$. The stick and slip parts of the motion are of similar time duration, as has been observed, for a much slower peeling speed, by Cortet et al. [10]. The front velocity reduces to below $0.5 \mathrm{~m} / \mathrm{s}$ in the primary stick regions, with formation of a characteristic thicker dark band [Figs. 1(b) and 2(b)] owing to the formation of cavitation bubbles and the expansion of entrapped air pockets [12].

During the slip phase we observe the rapid formation of regular fracture bands. These bands are not produced by a one-dimensional synchronized forward slip of the front, rather by transverse-moving fractures. Figure 2 shows close-up images of these bands and panel (a) points out one of the fracture tips, as it travels sideways at $\sim 300 \mathrm{~m} / \mathrm{s}$. The very large transverse speed of the fractures, only allows a thin dark band to develop before the next fracture sweeps by and detaches the tape again. We highlight that these fractures are not observed during regular low-speed peeling without slip, or during extended stick phases within the stick-slip regime.

These fracture bands are usually formed at an angle $\alpha$ [Fig. 1(b)] to the pulling direction, but the crack front can travel in either direction, i.e., have a component with or against the overall travel direction of the detachment front. Usually, the fracture starts at the leading edge of the front, therefore traveling most frequently backward, i.e., from left to right in Fig. 1(b), where the leading edge is on the left side. The sign of the angle $\alpha$ is of course random from one realization to the next. In isolated cases the fractures have even been observed to start at an interior point and travel in both directions. The thin dark line indicates the forward edge of the fracture marking a new contact line between the adhesive and the substrate, while the band from the previous contact is peeled off, by the rapidly moving transverse fracture front. The new contact locations experience strong tension and bubbles start forming and growing there, further highlighting their location $[12,13]$, before the next fracture arrives to detach the next band. Once the tape has peeled off the substrate, these dark bands dissipate, as the polymer readjusts and the cavitation bubbles shrink again, providing 


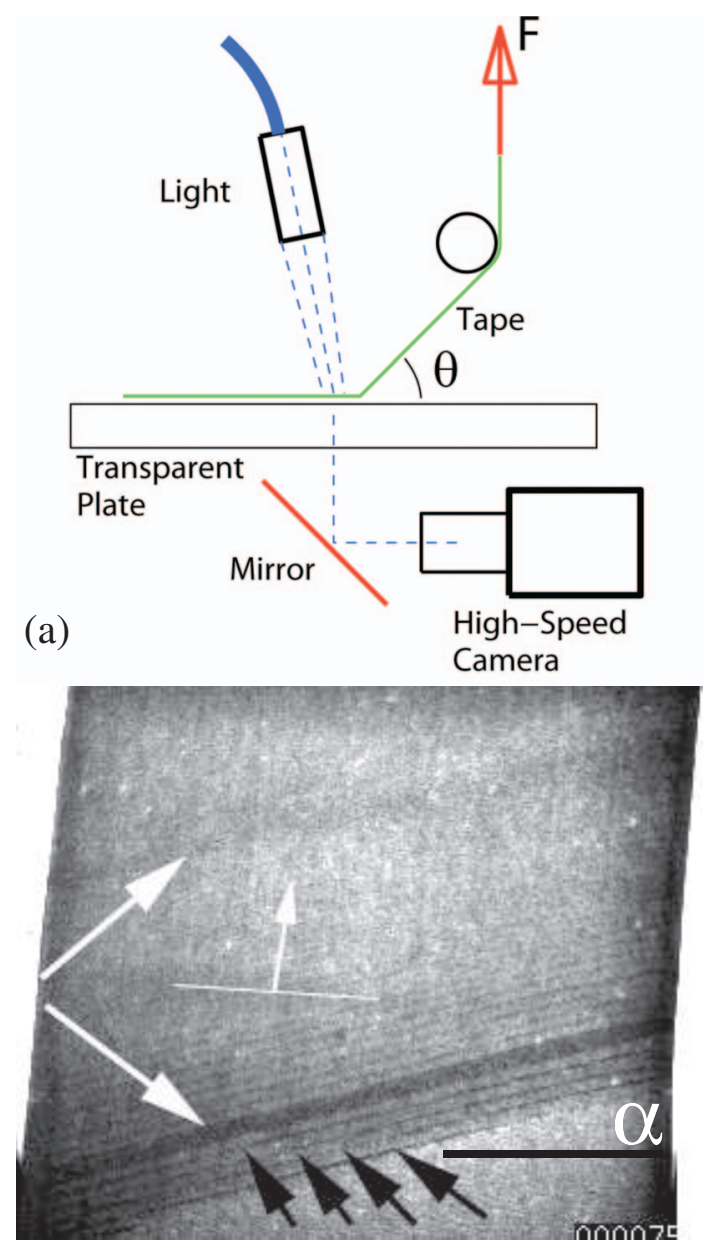

(b)

FIG. 1. (Color) Experimental setup and overall view of the peeling tape. (a) Sketch of the imaging setup. (b) Typical stickslip pattern formed during the rapid peeling of Scotch Tape, with the detachment front, i.e., where the tape separates from the substrate, having moved from top to bottom in the image. The thick dark bands (white arrows) are the primary stick locations, whereas the dark arrows point to the thin fracture bands, which appear during the slip phase. The thin white line indicates a wave-crest traveling in the detached tape. The dark bands dissipate after the tape detaches. The detached section also moves out of focus. The tape and image is here $12 \mathrm{~mm}$ wide. See also online video [23].

fewer scattering centers. The bands are therefore not as visible further along the detached part of the tape [Fig. 1(b)]. The detached part of the tape also moves quickly out of the focus. This is especially true for the highest frame rates and largest magnifications, where a larger aperture is needed for the imaging and the focal depth becomes quite narrow [14]. Note also that there is not a simple relationship between the transverse travel speed of the crack and the average front progression and/or angle $\alpha$, as there can be numerous cracktips moving at the same time, as shown in Fig. 2(c).

The visible fracture tip must connect back to the previous stuck line, but it travels parallel to the glass and moves too fast in a transverse direction to be directly observed, even at 1 Mfps in Fig. 3(a). It is smeared out during the $500 \mathrm{~ns}$

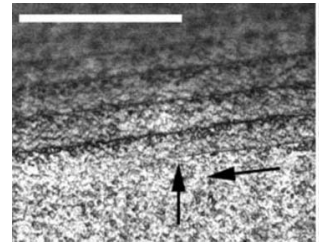

(a)

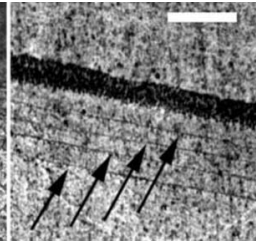

(b)

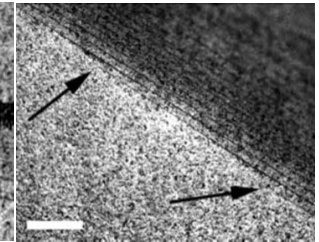

(c)
FIG. 2. Typical fracture bands. (a) Fracture front is moving to the left. The vertical arrow points at its tip. (b) The thick dark band is an earlier stick location, whereas the arrows point to thin lines which appear between the fractures during the slip phase. The region above the thick dark band did contain thin dark fracture bands which have dissipated during the stuck phase, which here lasted for about $1 \mathrm{~ms}$. (c) Two fractures traveling along the front. Scale bars are $1 \mathrm{~mm}$. The overall motions of the fronts are from top to bottom, with the lower parts still in contact with the glass plate. See also the online videos [23].

exposure, whereas the observable tip moves horizontally along the new contact line and its location is essentially stationary, thus making it clearly visible as the dark line. However, by taking intensity difference between video frames, the curved crack front becomes discernible, as shown in Fig. 3(b). Here we have calculated the intensity difference between the frame preceding the first frame in this sequence [Fig. 3(a)] and the individual frames which follow. It shows that the fracture front is curved between the two bands, as highlighted by the white broken line in one of the panels.

The fracture bands are quite uniform in width, measured perpendicular to the bands. However, the first band when the

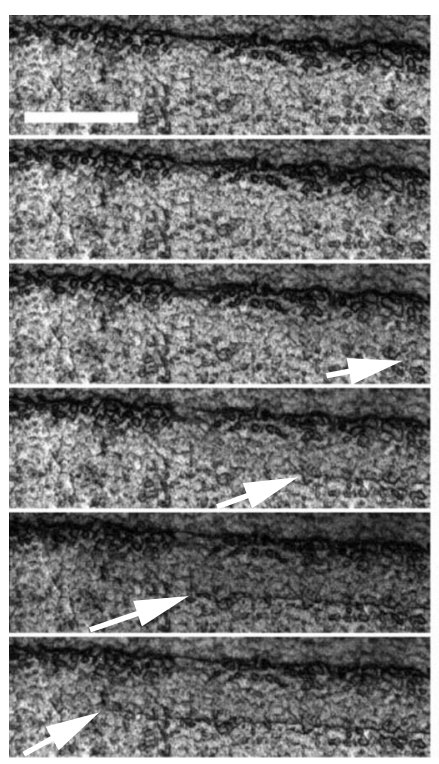

(a)

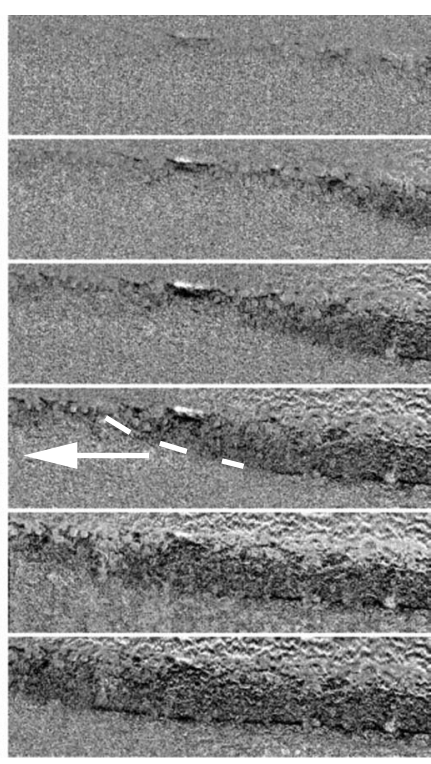

(b)
FIG. 3. Images of the progression of the fracture tip. (a) Frames taken from a 1 Mfps video sequence and the panels are separated by $1 \mu \mathrm{s}$. The left panels show the raw images, with the arrows pointing to the tip of the crack which is traveling to the left at about 325 $\mathrm{m} / \mathrm{s}$, for $\alpha \sim 2^{\circ}$. The overall motion of the front is downwards. The scale bar is $500 \mu \mathrm{m}$ long. (b) The difference in image intensity between the individual frames in (a) and the frame immediately preceding the sequence. See also video clip online [23]. 

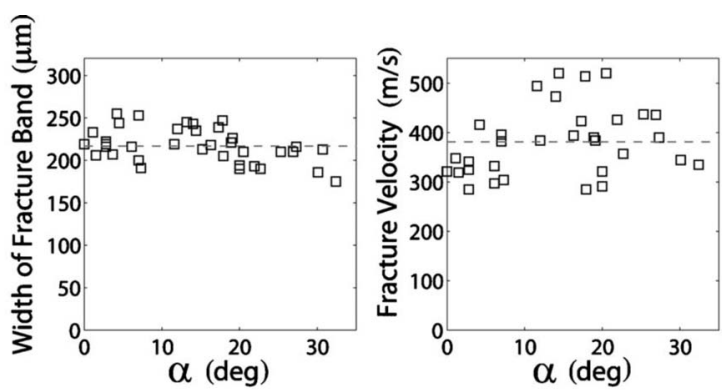

b
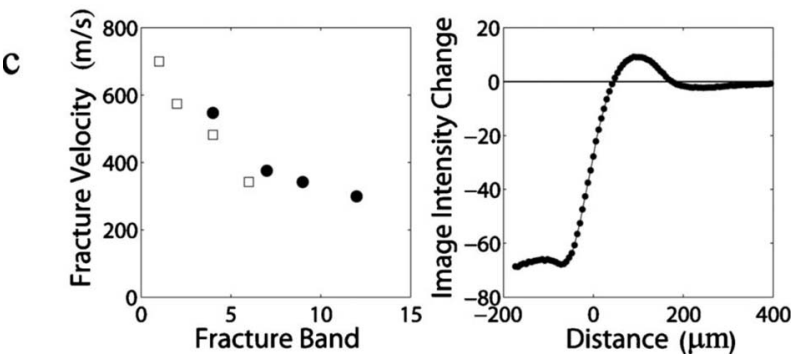

FIG. 4. Characteristics of the fracture bands. (a, b) The width and speed of the fracture bands, as a function of the angle of the front. Data exclude the first fracture bands, at start of slip phase. (c) Fracture velocity vs the number of the band from onset of slip. The symbols indicate two separate realizations. (d) The strain zone vs distance ahead of the detachment front. The differences in image intensity between a frame showing the undisturbed tape and the slowly moving front, at $3.5 \mathrm{~m} / \mathrm{s}$ during a long stick-phase. The averaging was performed both transverse over a $1.5 \mathrm{~mm}$ section from each frame, as well as over a sequence of 90 video frames. Compression of minute air pockets shows up as slight brightening in the image, highlighting the strain region in the adhesive which is responding to the applied pulling force.

front releases from a primary stick-zone, tends to be slightly wider $(\sim 20 \%)$, due to the large accumulated strain in the tape. We find that the width is not strongly dependent on the front speed, or the angle of the front $\alpha$, as is shown in Fig. 4(a).

Figure 4(b) shows that the fracture velocity, along the bands transverse to the motion of the front, also is not strongly dependent of $\alpha$, but has larger variation. We find that this velocity is fastest as the front releases from a stuck zone, as shown for two realizations in Fig. 4(c), but characterizing this is difficult with the limited image area and short duration of the video clips at the highest magnification and frame rates. This slowing in fracture speed is to be expected, from the effective reduction in the pulling force, through the rapid reduction in the strain in the tape, as the front travels through the slip phase. Once the tape has released enough of the built-up tension it sticks again. This being the basic dynamics underpinning the overall stick-slip phenomenon.

What determines the thickness of these fracture bands and their uniformity? The images reveal that the fracture is limited to the section of the tape which feels the advancing front. The applied tension is transmitted from the elastic film into the polymer adhesive which responds with localized longitudinal strain in the direction of the pulling, weakening the bonding with the substrate. It also pulls the film closer to the surface and locally compressing minute entrapped air

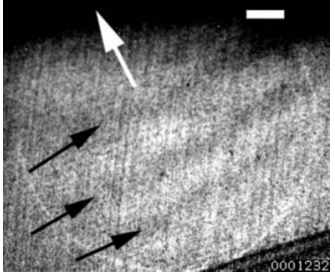

(a)

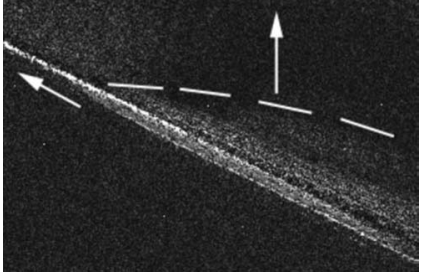

(b)
FIG. 5. Waves traveling in the detached tape. (a) Three wave crests (black arrows) traveling at $85 \mathrm{~m} / \mathrm{s}$ away from the front (dark region in the bottom right), in the direction of the white arrow. The scale bar is $1 \mathrm{~mm}$ and the exposure is $2 \mu \mathrm{s}$. (b) Image difference showing an isolated wave, traveling upwards in the detached tape, which is generated by a fracture front traveling to the left. See also online video [23].

bubbles, thus changing slightly the light transmitted through the tape. Figure 4(d) shows these intensity changes, following the front in the stuck region. This graph simply shows the extent of the region ahead of the front, which feels it approaching, but does not indicate the strength of these interactions. The bending stiffness of the tape may also play a role, as the fracture band is only four times the tape thickness.

The video clips, included online [23], show clearly that the crack front produces a well-defined wave which travels up the detached section of the tape, as shown in Fig. 5. The strongest waves are produced when the front dislodges from a stuck region, to begin the slip phase. The accompanying video shows that these waves move at a phase-speed $c$ $\sim 90 \mathrm{~m} / \mathrm{s}$ and a typical wavelength is $\lambda_{\text {tape }} \simeq 1.5 \mathrm{~mm}$. This gives a flapping frequency of $f=c / \lambda$ of $56 \mathrm{kHz}$, which is over the audible range. However, we expect that the primary stick-slip envelope encompassing a train of these waves will generate lower frequency $(\sim 2 \mathrm{kHz})$ audible ripping noise $[1,6]$. High-frequency acoustic sampling concurrent with the imaging, would be of interest to identify these higherfrequency components.

Our results are robust and qualitatively independent of both the application of the tape onto the substrate, as well as the exact peeling velocity, as long as it is very high. Keep in mind that while the manual pulling force is unknown we expect it to be approximately constant during the very short time of the observations $(<1 \mathrm{~ms})$, as it is produced by a rapid motion and inertia of a human arm. On the other hand, it is possible that the elasticity of the skin can allow rapid variations in the pulling force. However, if this were the case we would expect to see waves propagating toward the peeling front and not away from it. No waves were observed propagating from the pulling end toward the peeling front. In other words we believe neither the rapid switching from stick- to slip-phase nor the fractures themselves are produced by any sudden variations in the pulling force on $\mathrm{ms}$ time scale.

From a theoretical point of view the stick and slip phases are often identified by the intervals of increasing force and decreasing force respectively, for a fixed pull velocity. Since the tape is here manually driven, the pull velocity could fluctuate. This lack of control of the pull velocity can make 
changes in the pulling force feed into changes in the pull velocity. Therefore, a direct comparison of our experimental results with theoretical models of the stick and slip phases are not well defined [17].

The dynamics are not significantly affected by how strongly the tape is pushed onto the substrate, or even by our attempts to apply slight pretension to the tape by stretching it as it is spread onto the substrate. The horizontal angle of the front $\alpha$ [in Fig. 1(b)] also does not change the fracture phenomenon, even for angles as large as $30^{\circ}$. On the other hand, we find that the dynamics are strongly dependent on the vertical angle of the pulling force, $\theta$ in Fig. 1(a). The above results are for $\theta=45^{\circ}$ and are little changed for $\theta=34^{\circ}$. On the other hand, for $\theta=63^{\circ}$ the slip phase, or fractures, no longer appear.

It remains to be seen whether the observed substructure of fracture bands are of direct relevance to earlier experiments on the peeling of adhesives, which mostly focus on much slower peel velocities $[1,10]$. Further measurements (not allowed in the present setup) are needed to see whether any transverse fractures are present for these much smaller peel velocities. These bands could be intrinsic only to stick-slip peeling at the very fast peeling regime studied herein. The extensive review in Teisseire et al. [15] categorize the peeling in probe-tack experiments into fingering, cavitation and cracking, labeling large elastic deformation cracking as terra incognita. This region corresponds to large values of the nondimensional Maxwell fluid relaxation time $\mathbf{T}=V \tau / h_{o}$, where $V$ and $h_{o}$ are the characteristic velocity and dimension associated with the crack, whereas $\tau$ is the relaxation time of the glue. For our experiments $\mathbf{T} \sim 10^{6} \tau$, far into this unknown regime.

Lower frame-rate sideways imaging of tape peeling has shown 2 to $7 \mathrm{~mm}$ slip-zones (Fig. 14 in Ref. [10]). Keep in mind that at $350 \mathrm{~m} / \mathrm{s}$ the fracture will travel across the entire width of the $18 \mathrm{~mm}$ tape in $50 \mu \mathrm{s}$. It would therefore be entirely invisible to imaging at slower than $20 \mathrm{kfps}$. However, we suspect that peeling of tape from a rigid surface, differs in significant ways from experiments using the peeling from a roll. The pulling angle $\theta$ is in that latter case much larger and fluctuates in time. The elasticity of the substrate is also much higher, as the adhesive rests on another winding of the tape. Ciccotti et al. [1] report, in such a setup, a stable peeling for velocities over $3 \mathrm{~m} / \mathrm{s}$, accompanied by a soft hum. Our imaging is based on intense backlighting and cannot be applied to the peeling from a roll.

We have identified new aspects of the phenomenon which may help in modeling. Many theoretical models used to recreate the stick-slip dynamics, are based on one-dimensional models, ignoring variations in the transverse direction, which certainly are important at very high peel rates used herein. The most recent two-dimensional (2D) models predict the peel front breaking up into large number of small stuckpeeled segments $[6,16]$. Furthermore, the direct observations of the flapping waves in the detached part of the tape, might be incorporated into the current dissipation models which, in addition to the deformation in the adhesive glue, already incorporate acoustic dissipation, which depends on the slip rate and is proportional to the square of the local velocity [17].

Future experiments would benefit from simultaneous measurement of the pulling force in the tape, even though the individual fractures may be difficult to discern from the force variations. They could also be conducted under vacuum conditions, to ascertain the influence of entrapped microbubbles, which invariable become entrapped during the application of the tape onto the substrate. In addition, it would be of particular interest to see whether regular fractures are present during extremely fast pulling velocities $>30 \mathrm{~m} / \mathrm{s}$, where regular peeling would be expected to resume.

In summary, we have imaged a regular substructure of transverse fractures during the slip-phase of rapid peeling of adhesive tape. This new understanding might suggest strategies to design more efficient adhesives [18], or new materials to mitigate damage through fracture trapping $[19,20]$. Perhaps the repeatable alignment of these fracture bands can be exploited to more efficiently peel delicate microstructures during fabrication. These regular structures may also shed new light on triboluminescence, suggesting ways to produce more continuous X-ray emissions than are generated from peeling off a roll [9]. These stick-slip substructure may also be relevant to the sliding contact of two solids [21,22].
[1] M. Ciccotti, B. Giorgini, D. Vallet, and M. Barquins, Int. J. Adhes. Adhes. 24, 143 (2004).

[2] C. Gay and L. Leibler, Phys. Today 52(11), 48 (1999).

[3] C. Gay and L. Leibler, Phys. Rev. Lett. 82, 936 (1999).

[4] A. N. Gent and R. P. Petrich, Proc. R. Soc. London, Ser. A 310, 433 (1969).

[5] D. C. Hong and S. Yue, Phys. Rev. Lett. 74, 254 (1995).

[6] Rumi De, G. Ananthakrishna, Phys. Rev. Lett. 97, 165503 (2006).

[7] P. G. de Gennes, Langmuir 12, 4497 (1996).

[8] E. Lorenceau, F. Restagno, and D. Quere, Phys. Rev. Lett. 90, 184501 (2003).

[9] C. G. Camara, J. V. Escobar, J. R. Hird, and S. J. Putterman, Nature (London) 455, 1089 (2008).
[10] P.-P. Cortet, M. Ciccotti, and L. Vanel, J. Stat. Mech.: Theory Exp. 2007, P03005.

[11] T. G. Etoh et al., IEEE Trans. Electron Devices 50, 144 (2003).

[12] I. Chikina and C. Gay, Phys. Rev. Lett. 85, 4546 (2000).

[13] H. Lakrout, P. Sergot, and C. Creton, J. Adhes. 69, 307 (1999).

[14] S. T. Thoroddsen, T. G. Etoh, and K. Takehara, Annu. Rev. Fluid Mech. 40, 257 (2008).

[15] J. Teisseire, F. Nallet, P. Fabre, and C. Gay, J. Adhes. 83, 613 (2007).

[16] J. Kumar, M. Ciccotti, and G. Ananthakrishna, Phys. Rev. E 77, 045202(R) (2008).

[17] J. Kumar, R. De, and G. Ananthakrishna, Phys. Rev. E 78, 066119 (2008). 
[18] A. K. Geim et al., Nature Mater. 2, 461 (2003).

[19] T. Thomas and A. J. Crosby, J. Adhes. 82, 311 (2006).

[20] N. J. Glassmaker et al., Proc. Natl. Acad. Sci. U.S.A. 104, 10786 (2007).

[21] F. Heslot, T. Baumberger, B. Perrin, B. Caroli, and C. Caroli,
Phys. Rev. E 49, 4973 (1994).

[22] M. Urbakh, J. Klafter, D. Gourdon, and J. Israelachvili, Nature (London) 430, 525 (2004).

[23] See supplementary material at http://link.aps.org/supplemental/ 10.1103/PhysRevE.82.046107 for supplementary videos. 